\title{
Research Paper \\ Relationship between Visual Constructive Abilities and Activity of Daily Living in Home Dwelling Elderly Population
}

\author{
${ }^{*}$ Negin Chehrehnegar ${ }^{1}$, Fariba Keshavarzi ${ }^{1}$, Negar Rahnamaee ${ }^{1}$, Zahra Aghajafari ${ }^{1}$
}

1. Department of Occupational Therapy, School of Rehabilitation Sciences, Shiraz University of Medical Sciences, Shiraz, Iran.

Cftation: Chehrehnegar N, Keshavarzi F, Rahnamaee N, Aghajafari Z. [Relationship between visual constructive abilities and activity of daily living in home dwelling elderly population (Persian)]. Iranian Journal of Ageing. 2016; 11(2):220-225. http://dx.crossref.org/10.21859/sija-1102220

http://dx.crossref.org/10.21859/sija-1102220

Received: 2 March 2016

Accepted: 10 June 2016

Key words:

Aging, Activities of

daily living, Visual

constructive abilities

\section{AB STRACT}

Objectives Increasing life expectancy and decreasing birthrates have significantly contributed to an increased aging population throughout the world. This sudden change is a global phenomenon often resulting in biological changes that may have various consequences, such as reduced life power and coping skills in the elderly population. Cognitive deficits are one of the most severe impairments in the elderly people. Deficits in cognitive abilities, especially visual constructive skills, can have a considerable impact on the independency of the daily living skills of the elderly people. Self-care by individuals to maintain their life and wellbeing is a key element for their independency. The activity of daily living (ADL) can support personal life independency, and is considered as a morbidity index. In the present cross-sectional study, we assessed the visual abilities and ADL in older subjects to determine whether cognitive impairment is associated with changes in self-care behavior.

Methods \& Materials This study employed random sampling technique to select and recruit forty seven individuals aged between 60 to 80 years from Jahandidegan club in Shiraz, Iran. They were evaluated through "visual constructive ability" sub-scale from Loewenstein Occupational Therapy Cognitive Assessment (LOTCA) battery and "Katz Index", which were used to assess their associated skill and ADL, respectively. Data was collected through observation and interviews. Data analysis was performed through Pearson's correlation test using SPSS.

Results The mean age of the participants ( 9 women and 38 men) was $69.94 \pm 4.66$ years. Lower scores in cognitive domains predicted functional decline in some scales. There was a significant correlation between visual constructive ability and eating; however, no significant correlation was found between this sub-scale with bathing, moving, toileting, and bowel control.

Conclusion In summary, a significant correlation was noted between visual constructive abilities and dressing and eating activities. A weak correlation was observed between cognition and personal independency that may result from the less demanding cognitive processing tasks.

\section{* Corresponding Author:}

Negin Chehrehnegar, MSc.

Address: Department of Occupational Therapy, School of Rehabilitation Sciences, Shiraz University of Medical Sciences, Shiraz, Iran.

Tel: +98 (912) 6836728

E-mail: negin.chehrenegar@gmail.com 


\title{
رابطه مهارتهاى بينايى ساختارى و فعاليتهاى روزمره زندتى سالمندان مقيم جامعه
}

\author{
"نكين جهرهنكار'، فريبا كشاورزى'، نكار رهنمايى'، زهرا آقاجعفرى' \\ 1- كروه كاردرمانى، دانشكده توانبخشى، دانشكًاه علوم يزشكى شيراز، شيراز، ايران.
}

\begin{abstract}
حكSد

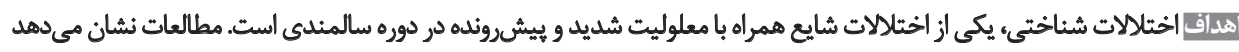

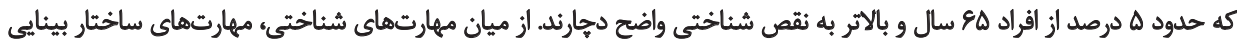

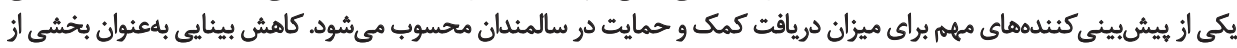

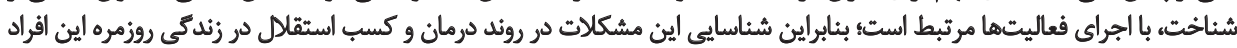
تأثير بسزايي دارد.

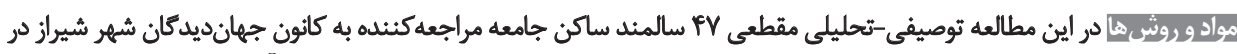

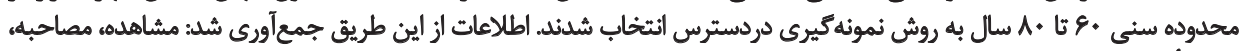

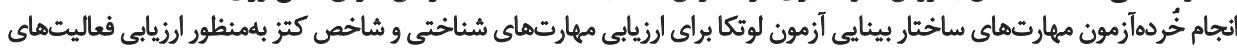

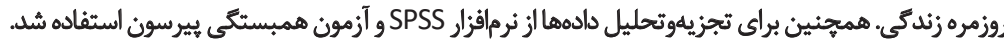

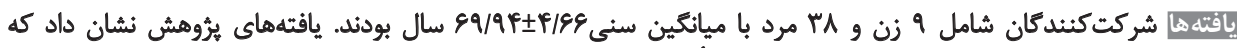

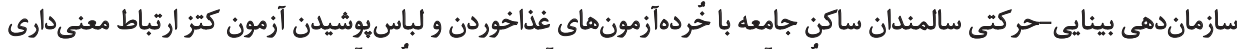

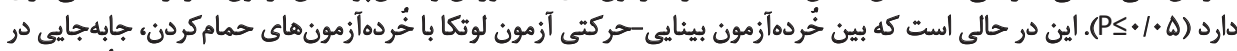

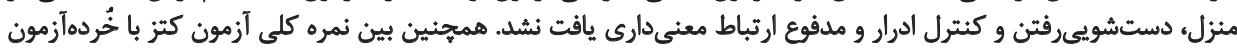

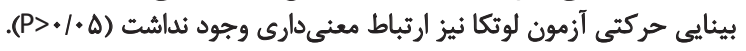

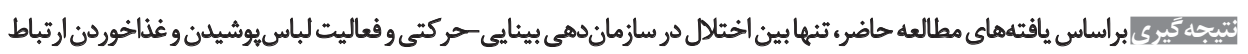

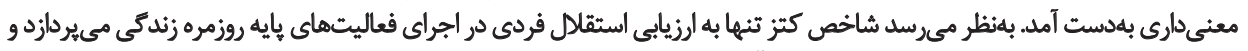

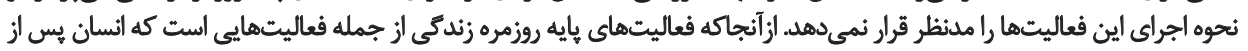

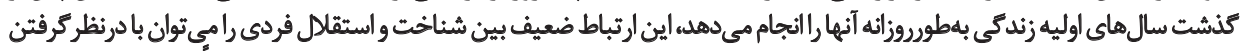

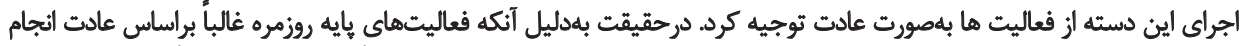

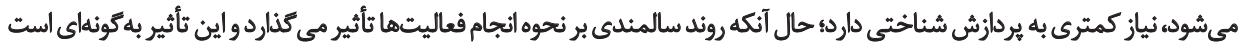

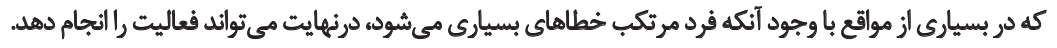

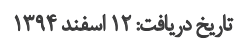

تاريخ يذيرش: ابr خرداد هوسا

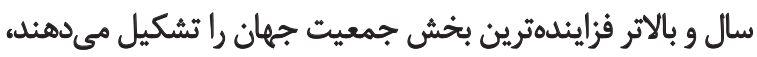

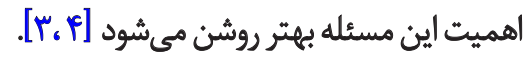

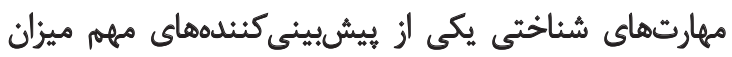

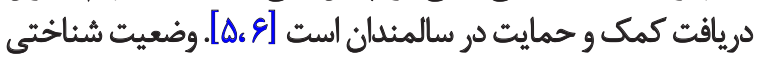

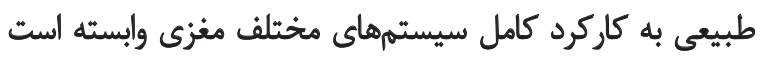

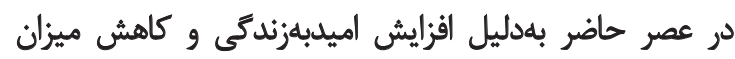

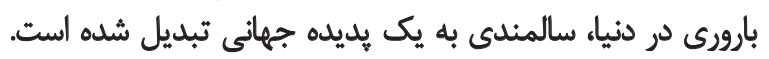

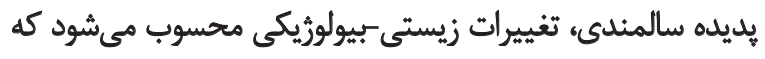

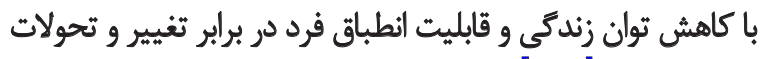

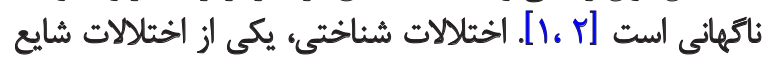

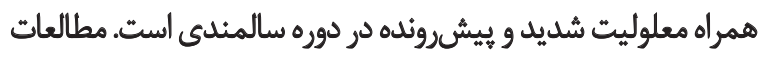

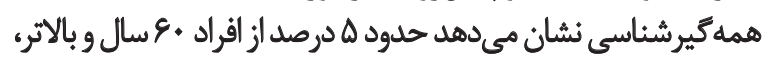


سالمندان مقيم جامعاه بررسى نشده است؛ بنابراين محققان يُروهش

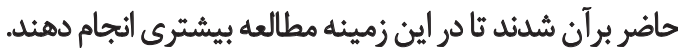

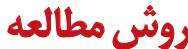

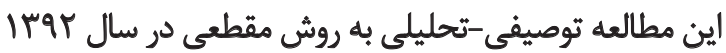

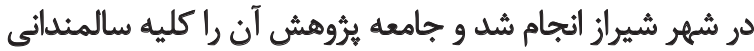

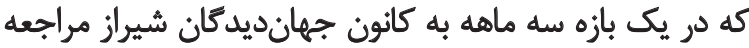

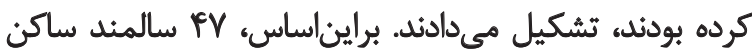

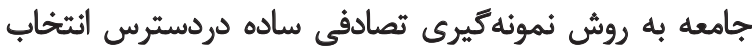

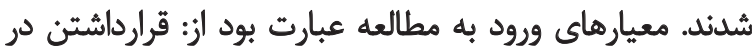

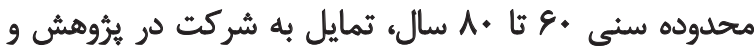

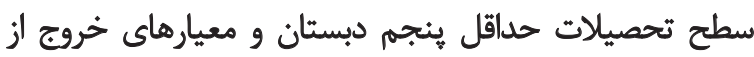

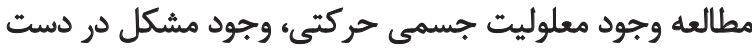

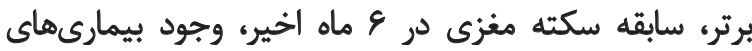

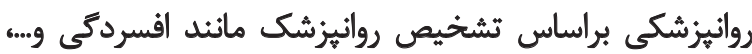

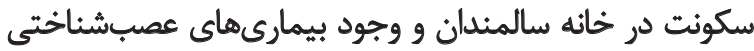

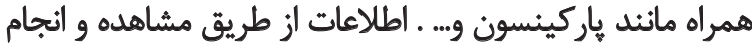

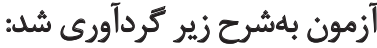

\section{آزمون ارزيابى شناختى لوتكا؟}

در ابتداى تحقيق از تمامى نمونههاى مورديثروهش رضايتنامه

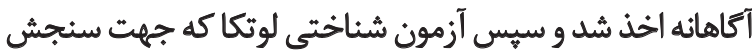

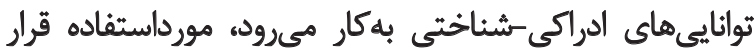

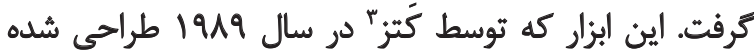

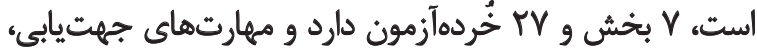

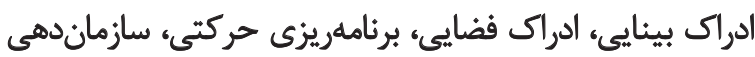

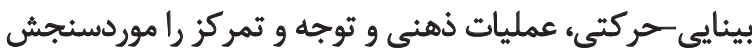

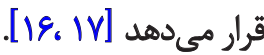

روايى نسخه فارسى ابزار موردنظر و همجينين تمام

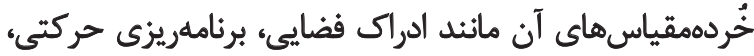

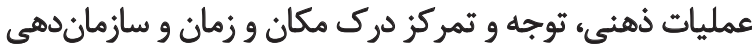

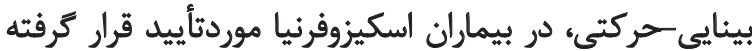

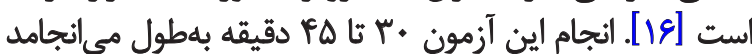

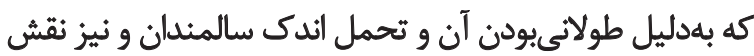

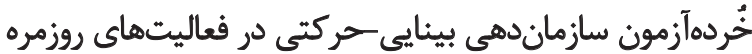

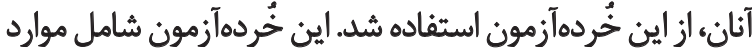

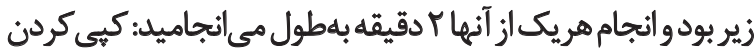

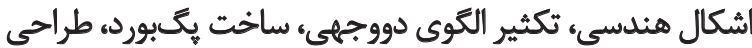

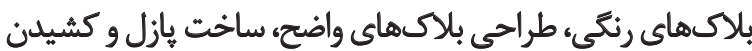

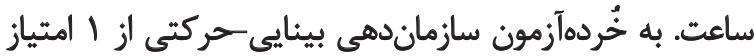
(يعنى بيمار قادر به انجام مهارت نيست) تا ها امتياز (يعنى

2. Lowen Stein Cccupational Therapy Cognitive Assessment 3. Katz
و با افزايش سن و تغييرات تحليلرونده و عملكردى كه در اين

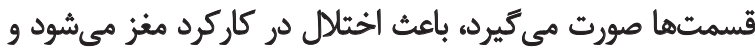

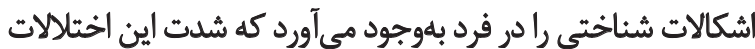

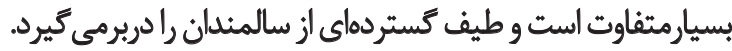
از حدود · · سالكى، تقريباً كاهش متغيرى در حداكثر توان

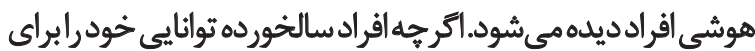

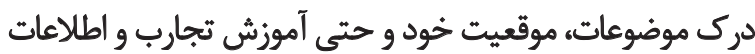

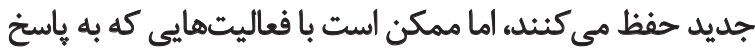

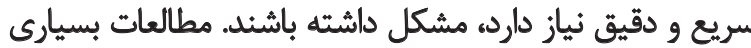

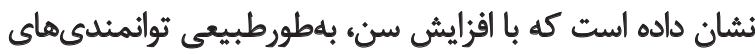

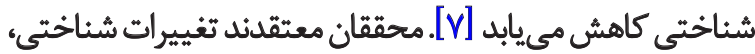

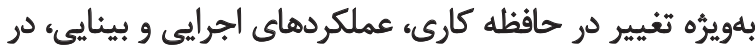

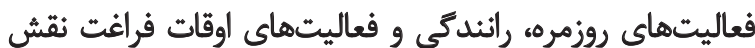

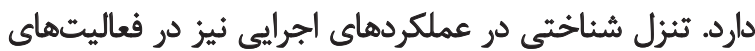

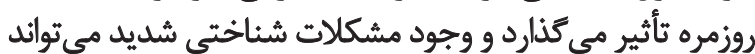

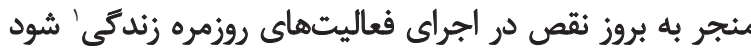

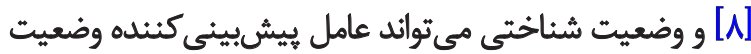

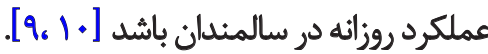
بلعنوان بخشى از شناخت، كاهش بينايى باجراي فعاليتهامرتبط

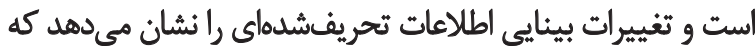

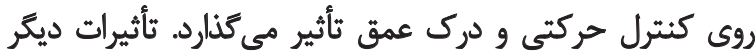

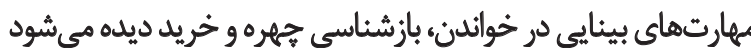

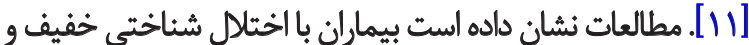

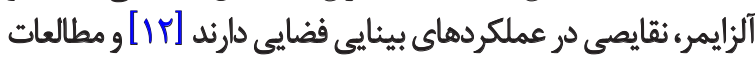

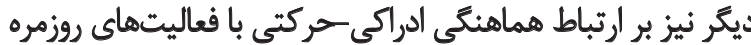

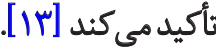

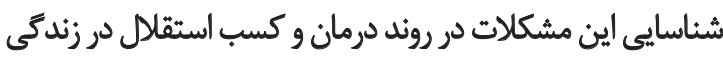

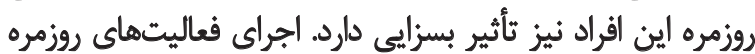

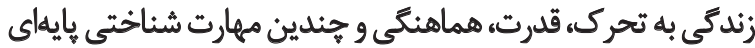

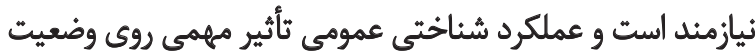

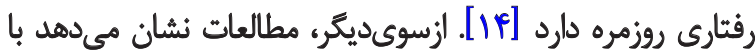

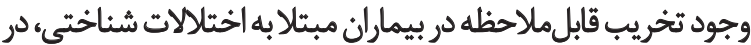

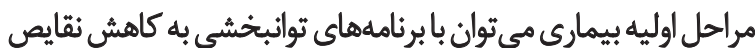

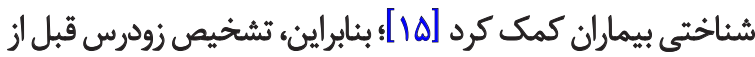

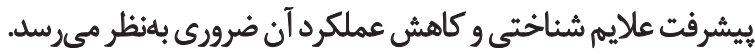
مطالعات مختلفى بلهورت جداكانه در مورد مشكلات شناختى

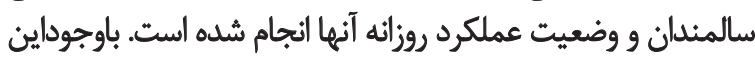

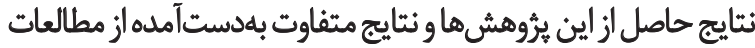

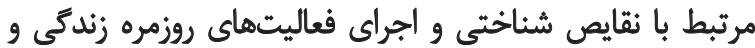

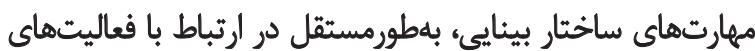

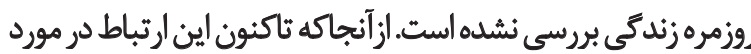

1. Activity of daily living 


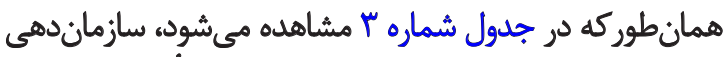

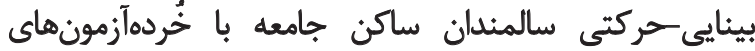

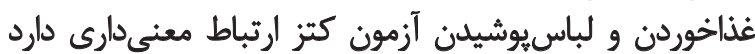

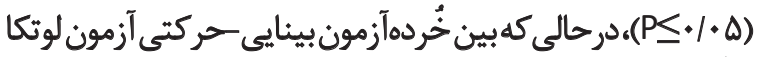

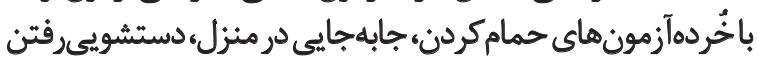

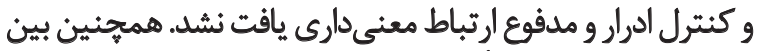

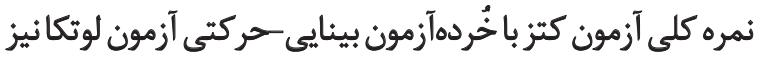

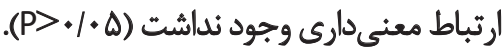

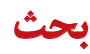

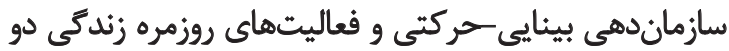

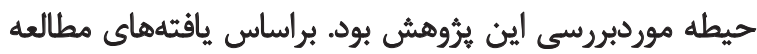

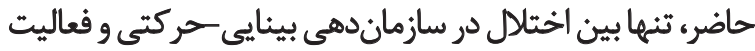

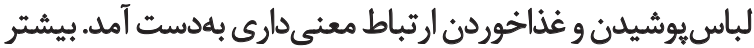

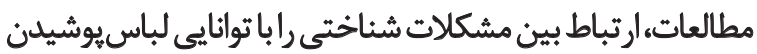

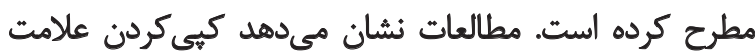

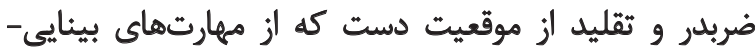

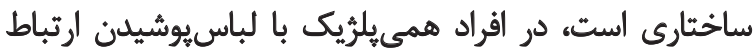

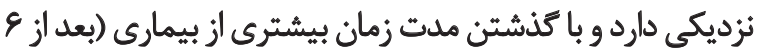

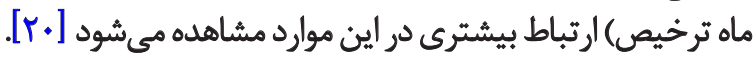
بلهعلاوهدرباره رابطه بين ناتوانى دربرنامهريزى بينايى حركتى

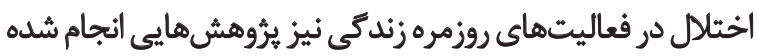

$$
\text { عملكرد مناسب متغير است) نمره داده مي شد. }
$$

براى ارزيابى فعاليتهاي روزمره زندكى سالمندان از آزمون

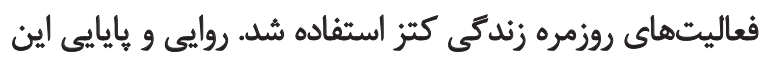

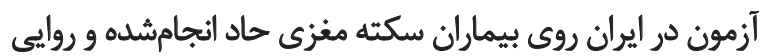

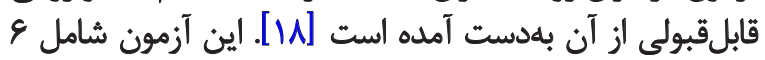

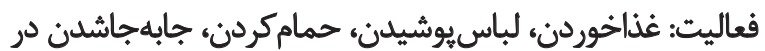

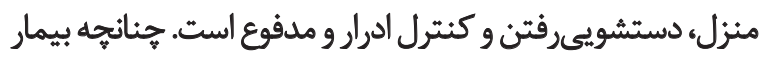

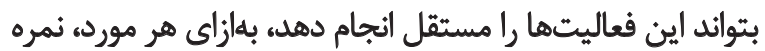

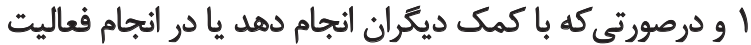

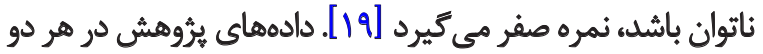

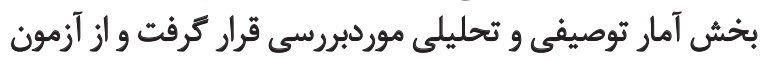
همبستكى ييرسون براى تجزيهوتحليل دادهايلها استفاده شد.

يافتهها

در اين مطالعه FV سالمند ساكن جامعه شهر شيراز شامل 9 زن زئن

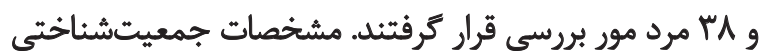

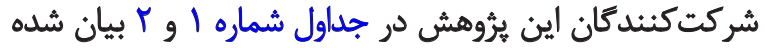

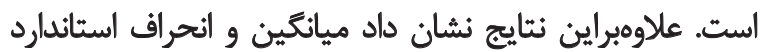

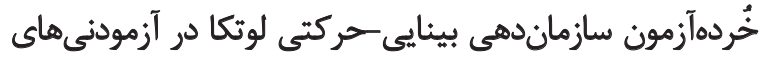

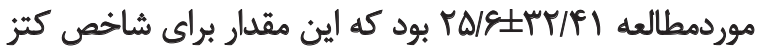
ه/VA بوده است.

جدول ا. مشخصات جمعيتشناختى سالمندان شركت كنئده.

\begin{tabular}{|c|c|c|}
\hline انحراف اسثاندارد & مياتخين & مثغير \\
\hline r/ge & $89 / 97$ & سن سن \\
\hline$r / F \Delta$ & $N F+N^{\prime}$ & سال هاى تحصيل \\
\hline
\end{tabular}

乩

جدول I. وضعيت شغلي سالمندان شركت كثنده.

\begin{tabular}{|c|c|c|}
\hline دوصد & فراواثى & هتغير \\
\hline$r \Delta / \Delta$ & ir & بيكار \\
\hline$r / 9$ & 10 & شاغُل \\
\hline Pr/s & r. & باز نشسته \\
\hline
\end{tabular}

L

جدول س. ضريب همبستكى خُردهآزمون بينايى حركتى آزمون لوتكا با خُردهآزمونهاي آزمون كتز (آزمون همبستكى بيرسون).

\begin{tabular}{|c|c|c|c|c|c|c|c|c|}
\hline نمره كلى أزمون كتيز & كثترل ادرار و مدفقو & دستشويحىرفتن & جابجايى در مثزل & حمامركرن & لباسيوشيدن & غذاخورن & & متغير \\
\hline.$M I f$ & - MOA &.$/ 11$ & $\cdot 1 \cdot+1$ &.$/ 11$ &.$/ T \Delta s$ &.$/ T \Delta$ & $r$ & خُردهاَّزمون \\
\hline.$/ I F A$ &.$/ 1 r^{\prime}$ & $\cdot|A T|$ & .1199 & $\cdot|A T|$ &.$/ .14$ & .1 .14 & $P$ & بينائيكا ركتى \\
\hline
\end{tabular}

几L 


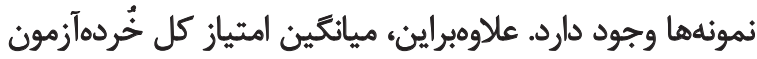

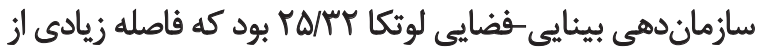

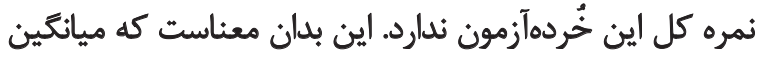

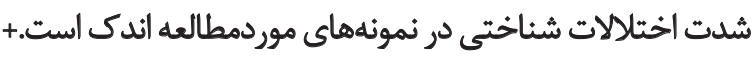

بيشنهادها:

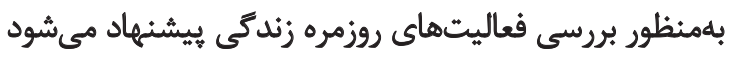

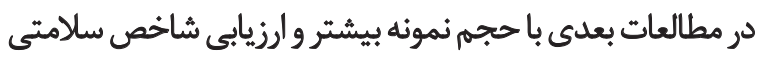

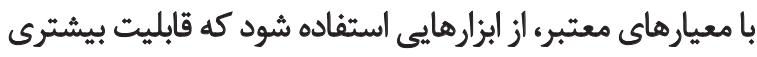
در تحليل فعاليتها داشته باشد.

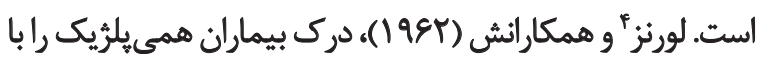

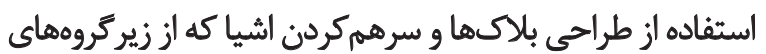

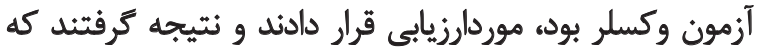

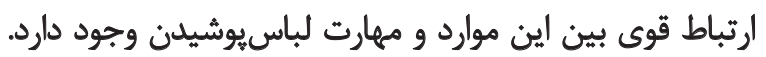

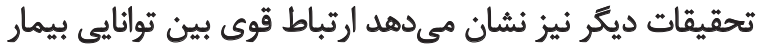

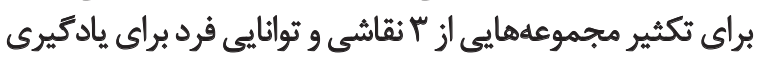

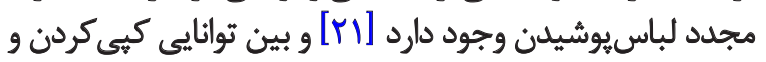

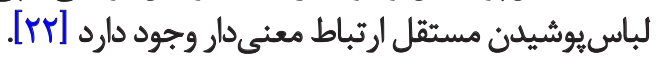

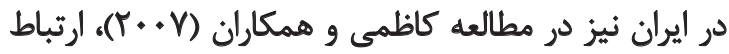

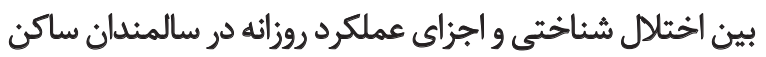

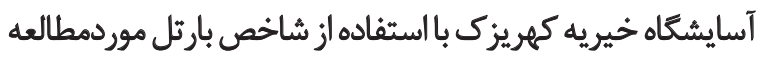

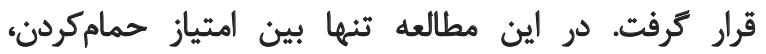

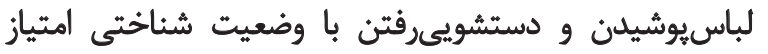

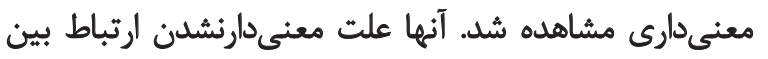

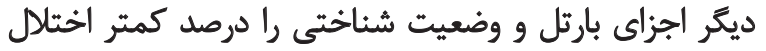

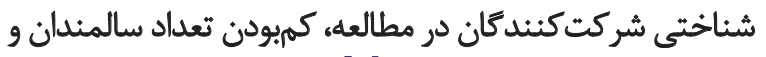

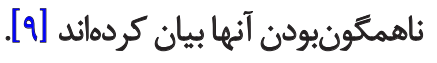

همجنين در مطالعهاى كه توسط اكبرى و همكارانش (1 + • (T)

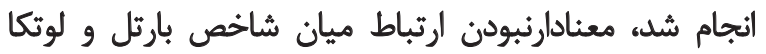

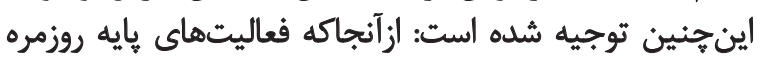

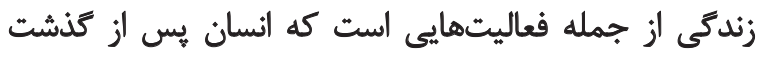

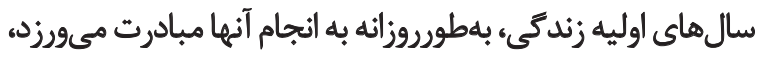

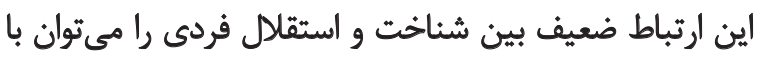

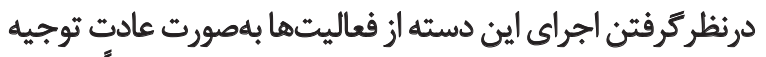

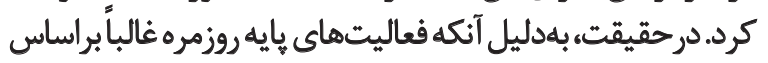

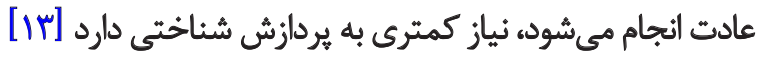

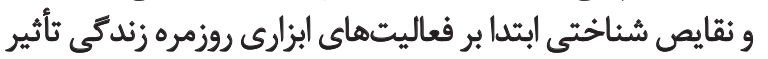

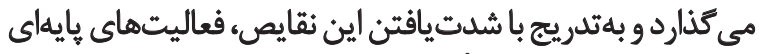

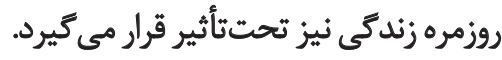

\section{نتيجه تيرى نثهايى}

مسئلهاي كه بيرامون ارتباط نمره كل شاخص كتر وخُخردها آزمون

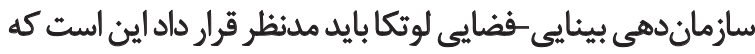

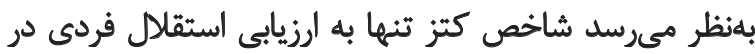

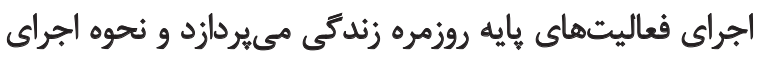

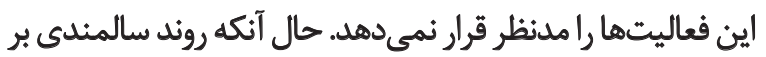

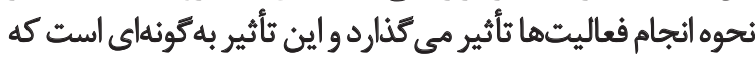

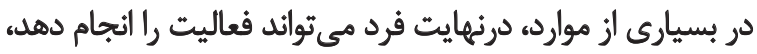

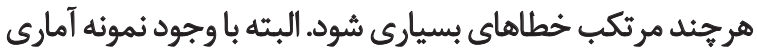

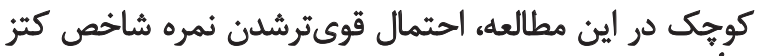
و خُردهآزمون سازماندهي بينايى -فضايى لوتكا با با افزايش تعداد 


\section{References}

[1] Delvarian M, Ghorbani Maftoon A. [Evaluation of nutrition in body mass index (BMI) of old age sanitarium of Shahroud (Persian)]. Paper presented at: Old Age Nutrition Congress; 2005 April 11-13; Tehran, Iran.

[2] Roukoei L, Kimiagar M, Ghafarpour M. [Old age nutrition in Tehran on 1997 (Persian)]. Tehran: Ashena Publication; 2006.

[3] Foroughan M. [Normalized elderly adults assessment examination in Iran (Persian)]. 1 $^{\text {st }}$ ed. Tehran: Arjmand Press; 2008.

[4] Rashedi V, Rezaei M, Gharib M. Prevalence of cognitive impairment in community-dwelling older adults. Basic \& Clinical Neuroscience. 2014; 5(1):28-30.

[5] Gill TM, Williams CS, Richardson ED, Tinetti ME. Impairments in physical performance and cognitive status as predisposing factors for functional dependence among nondisabled older persons. Journals of Gerontology Series A: Biological Sciences \& Medical Sciences. 1996; 51(6):283-88.

[6] Knopman DS, Kitto J, Deinard S, Heiring J. Longitudinal study of death and institutionalization in patients with primary degenerative dementia. Journal of the American Geriatrics Society. 1988; 21(9):47-56.

[7] Kamrani AA. [Memory \& cognition in aging (Persian)]. Tehran: University of Social Welfare \& Rehabilitation Sciences Publication; 2005.

[8] Titus MN, Gall NG, Yerxa EJ, Roberson TA, Mack W. Correlation of perceptual performance and activities of daily living in stroke patients. American Journal of Occupational Therapy. 1991; 45(5):410-18.

[9] Kazemi H, Ghasemi S, Sharifi F, Fakhrzade H, Ghaderpanahi M, Mirarefin M, et al. [Relation between elderly edult's cognitive status \& their functional capability in Kahrizak charity nursing home (Persian)]. Iranian Journal of Ageing. 2007; 4(12):16-25.

[10] Nazari H, Rashedi V, Mohammadi H, Yousefi M. [Relationship between cognitive status and activities of daily living among the elderly of nursing homes (Persian)]. Journal of Kermanshah University of Medical Sciences. 2015; 18(12):744-46.

[11] Farahbod M, Minaie A. [Adaption and standardization of the test of visual-motor skills revised (Persian)]. Journal of Rehabilitation. 2004; 5(1):39-48.

[12] Law LLF. Functional tasks exercise for older adults with cognitive impairment at risk of Alzheimer's disease. Townsville: James Cook University; 2013.

[13] Akbari S, Shafaroodi N, Akbarfahimi M, Ashayeri H, Kamali M. [Review of relation between cognitive status \& ADL independency rate in CVA people with right hand rehabilitation (Persian)]. Journal of Rehabilitation. 2009; 10(2):50-56.

[14] Zwecker M, Levenkrohn S, Fleisig Y, Zeilig G, Ohry A, Adunsky A. Mini-mental state examination, cognitive FIM instrument, and the loewenstein occupational therapy cognitive assessment: relation to functional outcome of stroke patients. Archives of Physical Medicine \& Rehabilitation. 2002; 83(3):342-45.

[15] Clare L, Woods RT. Cognitive training and cognitive rehabilitation for people with early-stage Alzheimer's disease: a review. Neuropsychological Rehabilitation. 2004; 14(4):385-401.
[16] Karbalaee Nouri A, Sadeghi A, Shamsolmaali Z. [Construct validity confirmation of Iranian version of Lowenstein Occupational Therapy Cognitive Assessment (LOTCA) (Persian)]. Journal of Rehabilitation. 2009; 10(2):33-37.

[17] Katz N, Itzkovich M, Averbuch S, Elazar B. Loewenstein Occupational Therapy Cognitive Assessment (LOTCA) battery for brain-injured patients: reliability and validity. American Journal of Occupational Therapy. 1989; 43(3):184-92.

[18] Mohamadinejad T, Azad A, Taghizadeh G, Mohamadian A. Persian version "Katz Independency Scale in ADL" test-retest raliability in Iranian person with acute CVA. Research in Rehabilitation Sciences. 2013; 9(4):10-17.

[19] Shelkey M, Wallace M. Katz index of independence in activities of daily living (ADL). Gerontologist. 1998; 10(1):20-30.

[20] Byorneby E, Reinvang I. Acquiring and maintaining self care skills after stroke. Scandinavian Journal of Rehabilitation Medicine. 1985; 17(5):75-80.

[21] Lorenze E, Cancro R. Dysfunction in visual perception with hemiplegia: Its relation to activities of daily living. Archives of Physical Medicine \& Rehabilitation. 1962; 43(4):514-17.

[22] Williams N. Correlation Between Copying Ability and Dressing Activities in Hemiplegia. American Journal of Physical Medicine \& Rehabilitation. 1967; 46(4):1332-340. 